\title{
A Broader Importance of NOPR to the Future of Nuclear Imaging
}

$\mathbf{I}_{\mathrm{n}}$ of The Journal of Nuclear Medicine (1), Hillner and coauthors once again emerge from the trove of data that is the $\mathrm{Na}$ tional Oncologic PET Registry (NOPR) with valuable insights. This time, the investigators turned their attention to 7 cancers, plus a hodgepodge of rare tumors, for which Medicare still does not cover the use of PET for restaging or suspected recurrence or for evaluating patients' response to treatment, except for PET facilities participating in Medicare's coverage-with-evidence-development (CED) program. The principal finding of Hillner et al. is that, in comparing data accrued during 2006-2009

\section{See page 831}

(the 2006 cohort) with data accrued after 2009 (the 2009 cohort), little has changed in how PET is applied to the clinical care of patients with these tumors or in the impact of PET on patient management. The authors conclude that given the large number of individuals studied $(30,911$ in the 2006 cohort; 54,747 in the 2009 cohort) and the stability of the results over time, there is little likelihood that further accrual will change the result. There is ample evidence to support Medicare coverage of the studied cancers for clinical indications related to restaging and suspected recurrence of cancer.

Received Mar. 5, 2012; revision accepted Mar. 9, 2012.

For correspondence or reprints contact: Bruce J. Hillman, 13129 Adona Lane, Wake Forest, NC 27587.

E-mail: bjh8a@virginia.edu

Published online Mar. 21, 2012.

COPYRIGHT (C) 2012 by the Society of Nuclear Medicine, Inc.

DOI: 10.2967/jnumed.112.104968
The development of the NOPR as a source of data and analysis that has been sufficiently trusted by the Centers for Medicare \& Medicaid Services (CMS) to help guide national coverage policy decisions is a model for how imaging professionals can responsibly have an impact on the care available to their patients. That data like these are available, substantial, and sufficiently directive to guide public policy is a testament to individual ingenuity and professional collaboration. Having played a peripheral role in helping forward the early development of the registry, I see NOPR's success as the result of a convergence of several factors: the demand for more rigorous, more generalizable research to demonstrate value warranting PET coverage; frustrating circumstances-specifically, spotty Medicare coverage for applying PET to the needs of cancer patients; the inventiveness of a handful of individuals who conceived and pursued the idea of NOPR, including Barry Siegel, Ed Coleman, and Brian Carey, later joined by Bruce Hillner, Tony Shields, and the Brown University statistical group, directed by Constantine Gatsonis; the availability of a research infrastructure, provided by the American College of Radiology Imaging Network, needed to put a resource of this magnitude into operation; and the organizational support of the Academy of Molecular Imaging, the owner of NOPR.

The current article by Hillner et al. propels us toward the end of the long road to achieving full coverage for using PET in cancer patients. How coverage for PET has been achieved is a cautionary tale that, given the current scrutiny of health care expenditures, bears important implications for future innovation. CMS considers coverage for new technologies on the basis of "medical necessity." Most such determinations are made by local
Medicare carriers (i.e., local coverage decisions). However, when considering new technologies such as PET, with broad applications, potentially high use, and major cost implications, CMS may choose to subject the technology to the more rigorous national coverage determination process, the outcome of which trumps any regional local coverage decisions. A national coverage decision is a hard row to hoe under any circumstances, but in addition, PET was among the first technologies assigned by CMS to make its way to coverage under CED.

CED is an old idea, newly applied. CED allows for provisional coverage of an otherwise noncovered procedure to promote the accrual of data to inform a coverage decision. To facilitate the process, CMS deems specific registries or clinical trials that it expects to provide the type and quality of data that will fulfill its needs. For the evaluation of PET and cancer, CMS deemed the NOPR (2). As such, providers participating in NOPR received payment for otherwise noncovered clinical PET examinations and contributed the required data for each subject to the NOPR database. With greater than $90 \%$ of U.S. PET facilities participating in NOPR, more than 200,000 patients, who otherwise would not have had access to the technology, received scans to further their diagnosis and treatment (1).

The progenitors of NOPR worked directly with CMS to determine what data would be necessary, if positive, to support broad coverage for using PET in the care of cancer patients. Such a negotiation is tricky business. Ideally, CMS, would have liked definitive evidence that using PET improves health outcomes by, for example, extending life or reducing the morbidity associated with the disease or treatment. This is a big hurdle for an 
imaging test, since imaging tends to be a single node in a chain of diagnostic and therapeutic maneuvers. It is difficult, time-consuming, and expensive to attribute improvements in health outcomes directly back to any single event in the chain, let alone a diagnostic imaging test (3). In recognition of these verities, CMS agreed to a study of therapeutic intent. Expanded coverage for PET would depend on the initiation of a national registry and the recruitment of institutions to supply data that would show whether using PET caused referring physicians to change their treatment plans for cancer patients.

Thus, the primary endpoint of the NOPR is change/no change in therapeutic decision making, a proxy for what CMS and we as good stewards of our patients' health and our national resources really want to know: for which cancers and which clinical indications does PET contribute to improved outcomes for cancer patients? Although we expect that there will be a strong correlation between decisions concerning therapy and patient health outcomes, the relationship is imperfect. This is all the more the case because of peculiarities in the NOPR design. Most significantly, the NOPR pre- and post-PET surveys measure intent, not what really occurred. Physicians being human and humans being what they are, physicians do not always do what they say they will, nor do patients always do what their physicians recommend. Second, the choice of change in management as the endpoint depends on the assumption that a change in management always benefits the patient. Clearly, this is not the case. Change in therapeutic intent as the primary endpoint for NOPR was an imperfect but necessarily practical solution to methodologic limitations, preventing the investigators from studying the true clinical impact of PET for cancer-related indications.
The saga of ${ }^{18}$ F-FDG PET coverage may be a bellwether for future nuclear medicine innovations, particularly broad-based ones with numerous potential applications. Practitioners attached to PET may have felt that the numerous small studies of PET and cancer conducted over the past $2 \mathrm{dec}$ ades provided sufficient evidence for PET coverage. However, more objective scrutiny of the published literature revealed important shortcomings. Specifically, research tended to be singleinstitutional and descriptive-attributes that make determinations of reliability and generalizability difficult. The latter of these is particularly important, since those who make decisions about paying for the clinical use of any new technology must be concerned with whether an innovation will work as well in broad community use as it does in the hands of highly specialized practitioners.

Because of escalating concerns over health care costs, future new technologies may have to cross 3 important hurdles to achieve Medicare and privatepayer coverage. Specifically, payers likely will require, first, clear and demonstrable benefit to patients. In ways noted above- the assumptions that intent reflected actuality and that all changes in therapeutic intent were beneficial- ${ }^{18}$ F-FDG PET got something of a pass that may not be extended to subsequent innovations. Second, payers likely will require demonstration of "value" - a defensible estimate of the cost per unit benefit. There is a dearth of such information with regard to PET and, for that matter, most other imaging modalities. Third, payers likely will require an estimate of the cost to American society of broad deployment of the technology. Given the concerns over the still-growing share of gross domestic product occupied by health care and its impact on national competitiveness, nuclear medi- cine and other imaging innovations may face the heretofore unimagined circumstance of providing good clinical benefit at a reasonable value but still experience difficulty in achieving coverage if deemed too costly.

The NOPR investigators are to be congratulated on their vision, energy, and persistence. Given the findings of the current article, it appears that there are few differences between the already-covered cancers and indications and those that continue to be not covered but paid for under CED. I agree with the authors' conclusion that "Even with less common cancers, the impact of PET on physician decision making for patient management seems remarkably uniform." It is time for CMS to cover these cancers for the studied clinical indications. The NOPR investigators can then turn their attention toward evaluating the more efficient sequencing of PET among the raft of imaging choices available to modern imaging practitioners.

\section{ACKNOWLEDGMENT}

No potential conflict of interest relevant to this article was reported.

Bruce J. Hillman

Department of Radiology

University of Virginia

Charlottesville, Virginia

\section{REFERENCES}

1. Hillner BE, Siegel BA, Shields A, et al. Impact of ${ }^{18} \mathrm{~F}$-FDG PET used after initial treatment of cancer: comparison of the National Oncologic PET Registry 2006 and 2009 cohorts. J Nucl Med. 2012;53: 831-837.

2. Hillner BE, Liu D, Coleman RE, et al. The National Oncologic PET Registry (NOPR): design and analysis plan. J Nucl Med. 2007;48: 1901-1908.

3. Hillman BJ, Gatsonis C. When is the right time to conduct a clinical trial of a diagnostic imaging technology? Radiology. 2008;248:12-15. 